\title{
GA-A23023 \\ STABILIZATION OF THE EXTERNAL KINK AND CONTROL OF THE RESISTIVE WALL MODE IN TOKAMAKS
}

\author{
by \\ A.M. GAROFALO, A.D. TURNBULL, E.J. STRAIT, M.E. AUSTIN, \\ J. BIALEK, M.S. CHU, E. FREDRICKSON, R.J. LA HAYE, \\ G.A. NAVRATIL, L.L. LAO, E.A. LAZARUS, M. OKABAYASHI, \\ B.W. RICE, S.A. SABBAGH, J.T. SCOVILLE, T.S. TAYLOR, \\ M.L. WALKER, and THE DIII-D TEAM
}

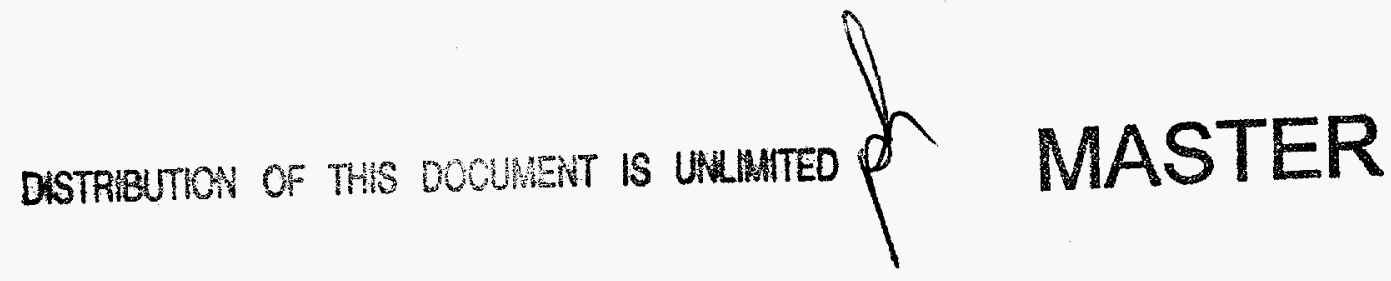

JANUARY 1999 


\section{DISCLAIMER}

\section{Portions of this document may be illegible in electronic image products. Images are produced from the best available original document.}




\title{
STABILIZATION OF THE EXTERNAL KINK AND CONTROL OF THE RESISTIVE WALL MODE IN TOKAMAKS
}

by

A.M. GAROFALO, ${ }^{1}$ A.D. TURNBULL, E.J. STRAIT, M.E. AUSTIN, ${ }^{2}$ J. BIALEK, ${ }^{1}$ M.S. CHU, E. FREDRICKSON, ${ }^{3}$ R.J. LA HAYE, G.A. NAVRATIL, ${ }^{1}$ L.L. LAO, E.A. LAZARUS, ${ }^{4}$ M. OKABAYASHI, ${ }^{3}$ B.W. RICE, ${ }^{5}$ S.A. SABBAGH, ${ }^{1}$ J.T. SCOVILLE, T.S. TAYLOR, M.L. WALKER, and THE DIII-D TEAM

This is a preprint of a paper presented at the 40th Annual Meeting, APS Division of Plasma Physics, November 16-20, 1998, New Orleans, Louisiana, and to be printed in Physics of Plasmas.

Work supported by U.S. Department of Energy under Grants DE-FG02-89ER53297 and DE-FG03-97ER54415, and Contracts DE-AC03-89ER51114, DE-AC02-76CH03073, DE-AC05-960R22464, and W-7405-ENG-48

\author{
1 Columbia University \\ 2The University of Texas at Austin \\ 3Princeton Plasma Physics Laboratory \\ 40 ak Ridge National Laboratory \\ 5Lawrence Livermore National Laboratory
}

GENERAL ATOMICS PROJECT 3466 JANUARY 1999 


\title{
Stabilization of the External Kink and Control of the Resistive Wall Mode in Tokamaks
}

\author{
A.M. Garofalo, ${ }^{\text {a) }}$ A.D. Turnbull, E.J. Strait, M.E. Austin, b) J. Bialek, ${ }^{\text {a) }}$ \\ M.S. Chu, E. Fredrickson, ${ }^{\text {c) }}$ R.J. La Haye, G.A. Navratil, ${ }^{\text {a) }}$ L.L. Lao, \\ E.A. Lazarus, ${ }^{\text {d) }}$ M. Okabayashi, ${ }^{\text {c) }}$ B.W. Rice, ${ }^{\text {e) }}$ S.A. Sabbagh, ${ }^{\text {a) }}$ J.T. Scoville, \\ T.S. Taylor, M.L. Walker, and The DIII-D Team \\ General Atomics, P.O. Box 85608 , \\ San Diego, California 92186-5608 U.S.A.
}

\begin{abstract}
One promising approach to maintaining stability of high beta tokamak plasmas is the use of a conducting wall near the plasma to stabilize low- $n$ ideal MHD instabilities. However, with a resistive wall, either plasma rotation or active feedback control is required to stabilize the more slowly growing resistive wall modes (RWMs).

Experiments in the DIII-D, ${ }^{1}$ PBX-M, ${ }^{2}$ and HBT-EP ${ }^{3}$ tokamaks have demonstrated that plasmas with a nearby conducting wall can remain stable to the $n=1$ ideal external kink above the beta limit predicted with the wall at infinity, with durations in DIII-D up to 30 times $\tau_{\mathrm{w}}$, the resistive wall time constant. More recently, detailed, reproducible observation of the $\mathrm{n}=1$ RWM has been possible in DIII-D plasmas above the no-wall beta limit. The DIII-D measurements confirm characteristics common to several RWM theories. The mode is destabilized as the plasma rotation at the $q=3$ surface decreases below a critical frequency of 1 to $7 \mathrm{kHz}$ ( $1 \%$ of the toroidal Alfvén frequency). The measured mode growth times of 2 to $8 \mathrm{~ms}$ agree with measurements and numerical calculations of the dominant DIII-D vessel eigenmode time constants, $\tau_{\mathrm{W}}$. From its onset, the RWM has little or no toroidal rotation $\left(\omega_{\text {mode }} \leq \tau_{\mathrm{w}}^{-1}<<\omega_{\text {plasma }}\right)$, and rapidly reduces the plasma rotation to zero.

Both DIII-D and HBT-EP have adopted the "smart shell" 4 concept as an initial approach to control of these slowly growing RWMs: external coils are controlled by a feedback loop designed to make the resistive wall appear perfectly conducting by maintaining a net zero radial field at the wall. Initial experiment results from DIII-D have yielded encouraging results.

a) Columbia University, New York, NY 10027.

b) The University of Texas at Austin, Austin, TX 87812.

c) Princeton Plasma Physics Laboratory, Princeton, NJ 08543.

d) Oak Ridge National Laboratory, Oak Ridge, TN 73831.

e)Lawrence Livermore National Laboratory, Livermore, CA 94551.
\end{abstract}




\section{Introduction}

For the Advanced Tokamak confinement concept to represent an economically attractive approach to commercial fusion power, operation at high $\beta_{\mathrm{N}}$ is essential in order to minimize size and cost of the power core, while at the same time maximizing the bootstrap current fraction with good alignment to permit steady state operation. The Negative Central Shear (NCS) Advanced Tokamak configuration 5,6 relies on stabilization of the low-n ideal magnetohydrodynamic (MHD) kink mode by a nearby conducting wall to achieve the required high values of $\beta_{N}$. (Here $\beta_{N}=\beta /(\mathrm{I} / \mathrm{aB}), \beta=2 \mu_{0}\langle\mathrm{p}\rangle / \mathrm{B}_{0}^{2},\langle\mathrm{p}\rangle$ is the volume averaged pressure, $\mathrm{B}_{0}$ is the external toroidal field at the radial center of the plasma chamber $\mathrm{P}_{0}, \mathrm{I}$ is the total toroidal current, and $\mathrm{a}$ is the plasma minor radius.)

While a perfectly conducting wall can, in principle, stabilize the kink mode, in a wall with finite conductivity the stabilizing image currents decay and the resulting unstable resistive wall mode (RWM) essentially leaks through the wall and grows with an exponential growth time of the order of the wall resistive decay time $\tau_{\mathrm{w}}$. Experimental work ${ }^{1-3,7-9}$ and theoretical calculations ${ }^{10-13}$ that included some form of plasma dissipation, have shown, however, that the plasma can be stabilized for times much longer than $\tau_{\mathrm{w}}$ if the plasma rotation rate $\Omega_{\mathrm{p}}$ is sufficiently fast relative to the wall. With plasma rotation and dissipation the ideal external kink now branches into two modes: (1) a plasma mode, that rotates at speed $\omega \sim \Omega_{\mathrm{p}}$ and is wall stabilized by continually regenerating the image currents for $\Omega_{\mathrm{p}}>\tau_{\mathrm{w}}^{-1}$, and (2) a RWM that is nearly stationary with respect to the wall $\left(\omega \sim \tau_{\mathrm{w}}^{-1} \ll<\Omega_{\mathrm{p}}\right)$ and can also be completely stabilized if the plasma rotation rate $\Omega_{p}$ is greater than some critical value $\Omega_{\mathrm{c}}$.

In recent DIII-D experiments, reproducible wall stabilized plasmas with enhancement factors $E_{\mathrm{w}}=\beta_{\mathrm{N}} / \beta_{\mathrm{N}}^{\text {nowall }}$ exceeding the previous maximum ${ }^{1,7-8}$ and lifetimes $\tau_{\mathrm{L}}$, during which wall stabilization was maintained, exceeding the previous duration by a factor of 3 , have been achieved using newly developed operational techniques. (Here, $\beta_{N}^{\text {no wall }}$ is the $\beta_{N}$ limit predicted without wall stabilization.) The slowing of the plasma rotation noted in earlier DIII-D experiments ${ }^{7}$ is shown to be strongly correlated with $E_{w}$ exceeding unity and persists while $\beta_{\mathrm{N}}>\beta_{\mathrm{N}}^{\text {no wall }}$ even in the absence of any detectable MHD activity. The critical rotation for stabilization $\Omega_{\mathrm{c}}$ is found to be robustly reproducible for repeated plasma conditions but is dependent on plasma conditions. Improvements in diagnostics capability and reproducibility of the discharges have furthermore made a direct identification of the RWM possible; the mode structure is measured here from electron cyclotron emission (ECE) spectroscopy and is compared directly to numerical predictions of the RWM structure.

Two promising avenues for stabilizing the RWM are being considered: active control of the plasma rotation, and active feedback using magnetic coils. A 3D electromagnetic code, VALEN, has been developed to model active feedback control of the RWM in a tokamak. 
Code predictions and initial experimental tests of active control of the RWM in the DIII-D and HBT-EP tokamaks show encouraging results toward the feasibility of ultimately controlling the RWM growth. 


\section{Passive Stabilization of the External Kink}

Discharges exceeding the $\beta_{\mathrm{N}}$ limit to $\mathrm{n}=1$ external kink calculated in absence of a wall can now be obtained reproducibly using a new technique in DIII-D. An early current ramp with some neutral beam heating is followed by a short constant current period during which the plasma is rapidly heated to high $\beta$ and a transition to $\mathrm{H}$-mode is triggered. This is then followed by a second current ramp to lower the internal inductance to $\ell_{\mathrm{i}} \sim 0.7$. The new technique produces single null divertor NCS target plasmas with low edge safety factor $\mathrm{q}$, and broad pressure and current density profiles that are coupled strongly to the wall. 5,6 $\mathrm{E}_{\mathrm{W}}>1$ is routinely achieved before other instabilities disrupt or degrade the target plasma.

Using this technique, the enhancement factor $E_{\mathrm{w}}$ has been extended to $E_{\mathrm{w}} \sim 1.4 \pm 0.05$ in discharge \#92544. The time history is shown in Fig. 1(a). $E_{W}$ exceeds unity in this discharge for a period of $\sim 200 \mathrm{~ms}$, which is a factor 3 greater than the previous duration reported in Refs. 1,7-8. At the time that this discharge reaches its maximum $\beta_{\mathrm{N}}$, GATO code $^{14}$ calculations show it is strongly unstable to an ideal kink ballooning mode with no wall stabilization, but near marginal stability with a perfectly conducting wall at the position of the DIII-D vacuum vessel (see Fig. 2). The results of the GATO stability calculations are found to be insensitive to variations in the equilibrium that are consistent with the discharge diagnostics, using the same approach as described in Ref. 7. In particular, the GATO results are not sensitive to the uncertainty in the edge current density.
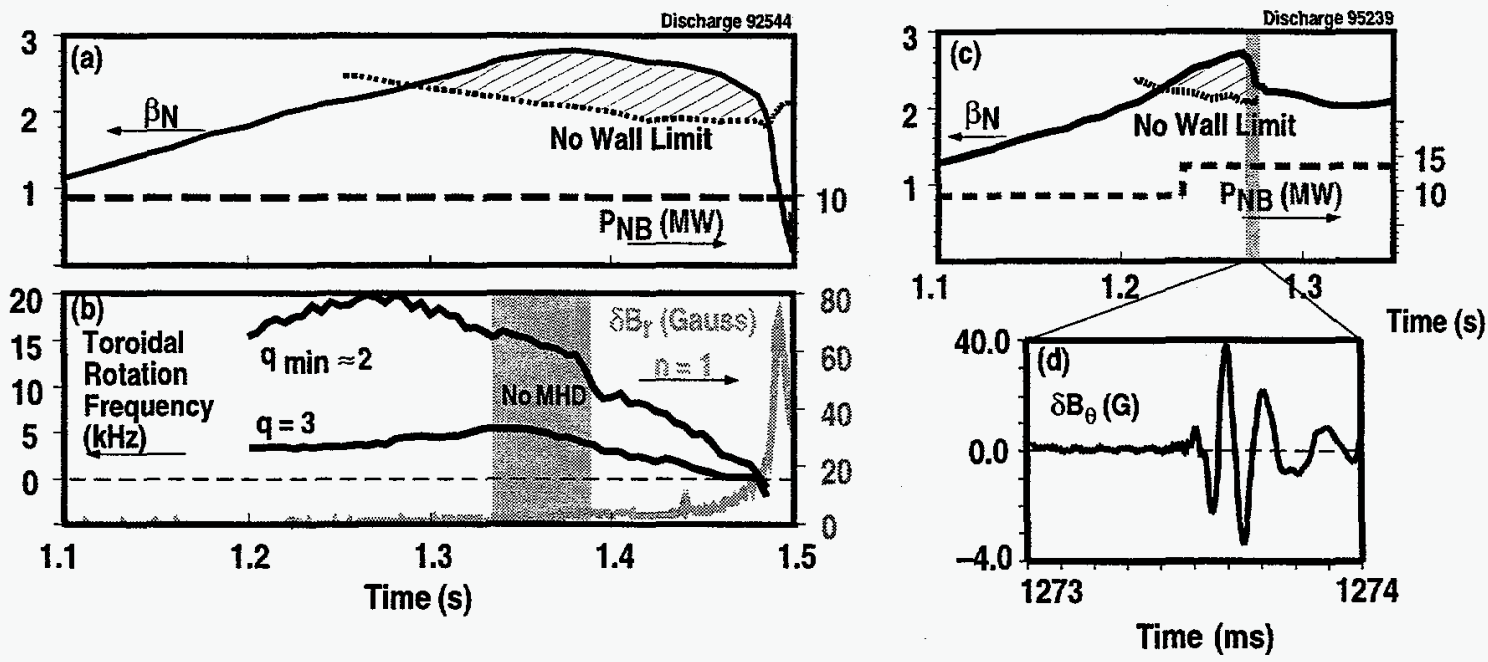

Fig. 1. Comparison of time evolution and termination of the high beta phase for discharges near the ideal wall stability limit. (a), (c) Normalized beta and neutral beam power. (b) Plasma rotation frequency at two radial locations, and the $\mathrm{Br}$ amplitude of the slowly growing, slowly rotating RWM measured from saddle loops outside the vessel in discharge \#92544. (d) Bq amplitude of the rapidly growing, rapidly rotating ideal kink-ballooning mode in discharge \#95239. 


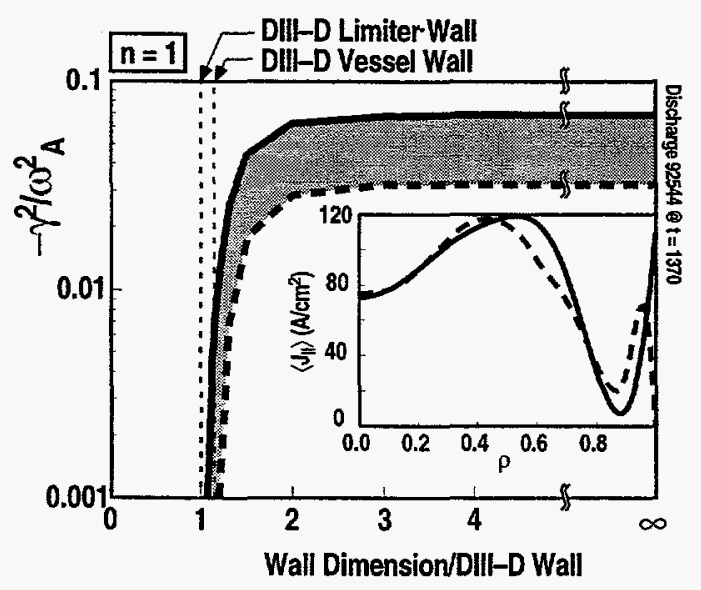

Fig. 2. Calculated growth rate of the $n=1$ ideal kink mode normalized to the Alfvén frequency for discharge \#92544 at the time of maximum beta $(t=1.37 \mathrm{~s})$. Solid curve shows results for an equilibrium reconstruction allowing finite flux averaged toroidal current density $\left\langle\mathrm{J}_{\phi}\right\rangle$ at the edge. Dashed curve is for case with $\langle\mathrm{J} f\rangle=0$ at the edge. Inset shows the profiles of $\left\langle\mathrm{J}_{\phi}\right\rangle$ for the two cases.

A two mode analysis of measurements of the time response of the vessel finds that the slowest $n=1$ eigenvalue has a time constant of $\tau_{\mathrm{w}}=7 \mathrm{~ms}$. This is confirmed by estimates from the VALEN 3D model for the DIII-D vessel and coil system, which predict $\tau_{w}=5.8 \mathrm{~ms}$. Wall stabilization was therefore maintained in discharge \#92544 for about 30 wall times.

The high beta phase of the discharge ends with an $n=1$ mode which has the characteristics predicted for a resistive wall mode: the mode growth starts as the plasma rotation decreases; the growth time of about $8 \mathrm{~ms}$ matches well the wall time constant $\tau_{\mathrm{w}}$ and the mode rotation $\omega$ is $\sim 60 \mathrm{~Hz}$ from the mode onset, consistent with the predicted RWM rotation $1 / 2 \pi \tau_{\mathrm{w}} \sim 40 \mathrm{~Hz}$. Comparable results have been obtained in a number of discharges with similar conditions.

In contrast, a similar discharge, \#95239 at about the same maximum $\beta_{\mathrm{N}}$ but with greater heating beam power shows an $n=1$ ideal mode growing on the Alfvénic time scale of about $40 \mu \mathrm{s}$, causing a beta drop [Fig. 1(c)]. The rapid growth of the mode and rapid rotation of mode is shown in Fig. 1(d). These observations are consistent with stability calculaions that show \#92544 is near marginal stability assuming a perfect conducting wall at the DIII-D vessel location. 


\section{Plasma Rotation in Wall-Stabilized Plasmas}

It is consistently observed that the plasma rotation slows when $\beta_{\mathrm{N}}$ exceeds the calculated no wall limit [Fig. 1(a), for example]. The slowdown is not correlated with any observable MHD activity, although the presence of MHD activity can sometimes accelerate the slowdown. The time histories of $\mathrm{d} \Omega / \mathrm{dt}$ are shown for several different discharges in Fig. 3 versus $\beta_{N} / \beta_{N}^{\text {nowall }}$. The correlation with $E_{W}>1$ is clear; when $E_{w} \sim 1$ the sign of $d \Omega / d t$ changes in each case. Possible MHD causes of the slowdown include the electromagnetic drag from a small amplitude, undetected RWM, or the electromagnetic drag from the internal continuum resonances of the wall-stabilized RWM. ${ }^{7}$ In either case, the RWM itself is most likely associated with the slowdown.

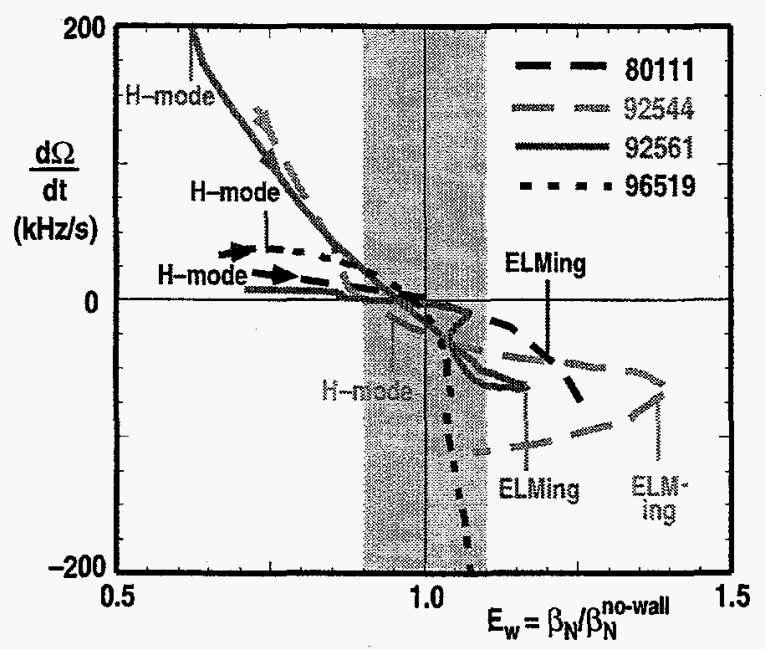

Fig. 3. Correlation of the rotation slowing rate $d \Omega / d t$ at a normalized minor radius $\rho=0.55$ for several wall stabilization discharges versus enhancement factor $E_{W}$. The $H$-mode transitions, ELMing, and other MHD activity onset times are also indicated.

The slowdown of the plasma rotation below a critical rotation rate $\Omega_{c}$ in a discharge with $\beta_{\mathrm{N}}$ above the no wall limit leads to an unstable RWM. The value of $\Omega_{\mathrm{c}}$ is found to be robustly reproducible for repeated plasma conditions, but is dependent on plasma conditions. For discharge \#92544 in Fig. 1, $\Omega_{c}$ evaluated at the $q=3$ surface is about $1 \mathrm{kHz}$, in agreement with previous DIII-D observations. 1,8 A considerably higher value of $\Omega_{\mathrm{c}}$ was reproducibly determined for a different set of discharges during a magnetic braking experiment. In this experiment a small static external $n=1$ field is applied ${ }^{8}$ using the error field correction coil (C-coil), to brake the plasma rotation. Figure 4 shows the results for three representative discharges with the same plasma conditions, but with varying braking current. ${ }^{15}$ A stronger braking produces an earlier deceleration of the $q=3$ surface and results in earlier onset of the RWM. In each case, however, the RWM appears when the rotation frequency at the $q=3$ surface is $6.5 \pm 0.5 \mathrm{kHz}$. The discharges of Fig. 4 just 


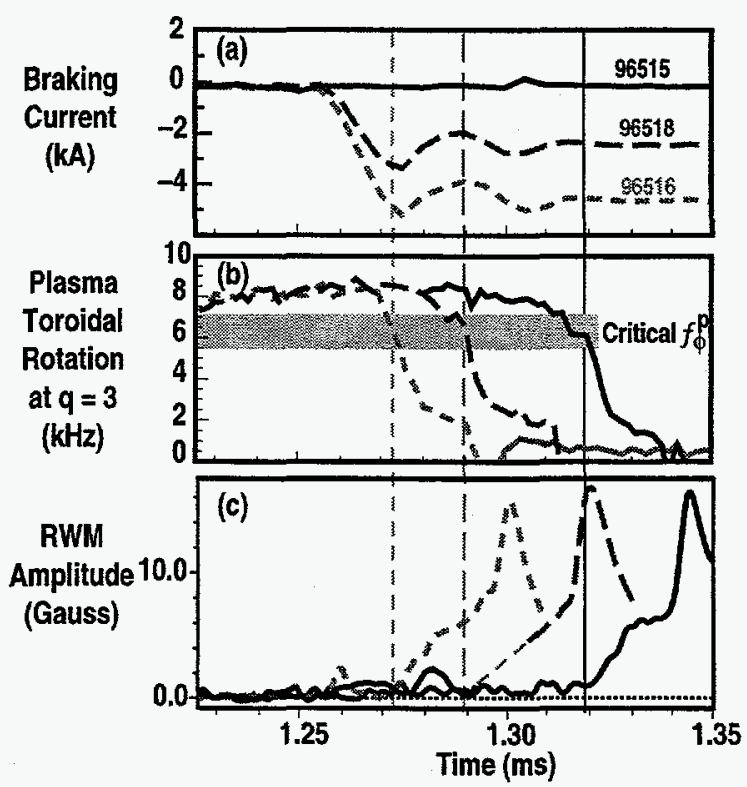

Fig. 4. Controlled variation of the RWM onset through C-coil braking of the plasma rotation in similar discharges. Time evolution of (a) $\mathrm{C}$-coil current, proportional to the magnetic braking field; (b) toroidal plasma rotation at the $\mathrm{q}=3$ surface; and (c) $\mathrm{B}_{\mathrm{r}}$ amplitude of the RWM. The vertical lines denote the onset of the RWM, corresponding in each discharges to a $q=3$ rotation of about $6.5 \mathrm{kHz}$.

slightly exceed the no wall limit, and the predicted RWM structure has an edge peeling structure, unlike discharge \#92544 for which $\mathrm{E}_{\mathrm{w}}$ significantly exceeds 1 , and the predicted mode structure is more global. These differences suggest two plausible explanations for the variation in the value of $\Omega_{\mathrm{c}}$ : (1) theory and modeling have suggested that to stabilize the RWM when $\beta$ is just above the no wall limit requires a more rapid plasma rotation than at somewhat higher $\beta, 16$ and (2) in discharges where the predicted RWM structure has larger internal poloidal components the RWM may couple more strongly to the rapid rotation of the core region, therefore requiring for stabilization a lower plasma rotation at the $q=3$ surface.

The experimental observations of $\Omega_{\mathrm{c}}=2 \pi f \sim 10^{4} \mathrm{~s}^{-1}$ disagree with predictions of ideal MHD theory that include dissipation only by resonance with the Alfvén waves, ${ }^{17} \Omega_{\mathrm{c}} \sim \tau_{\mathrm{A}}^{-1}$ $>10^{6} \mathrm{~s}^{-1}$, where $\tau_{\mathrm{A}}$ is the poloidal Alfvén time. The observations also disagree with resistive MHD theories that require formation of islands for RWM stabilization at low rotation, ${ }^{12,13}$ $\Omega_{\mathrm{c}} \sim \tau_{\mathrm{w}}^{-1} \leq 3 \times 10^{2} \mathrm{~s}^{-1}$. The observations are in somewhat better agreement with predictions of ideal MHD theories that include dissipation through toroidal coupling of the mode to sound waves, ${ }^{10} \Omega_{\mathrm{c}} \sim 0.05 \tau_{\mathrm{A}}^{-1} \sim 10^{5} \mathrm{~s}^{-1}$. 


\section{Resistive Wall Mode Identification in a Rotating Plasma}

The RWM is a mode that grows while nearly stationary with respect to the wall as the plasma is rotating: $\omega \sim \tau_{\mathrm{w}}^{-1} \ll \Omega_{\mathrm{p}}$. Figures 5(a)-(c) show a clear example of this characteristic. In contrast to the RWM case, Figs. 5(d)-(f) show a typical example of a locked tearing mode (LTM). At both the RWM onset and the LTM onset, an $n=1$ radial field essentially stationary with respect to the wall is seen to slowly penetrate through the wall [see Figs. 5(a) and 5(d)]. However, at the time of the RWM onset, the plasma rotation, measured from CER spectroscopy, is faster than $4 \mathrm{kHz}$ over nearly the entire minor radius. It is after the onset of the RWM that the plasma rotation decreases rapidly to zero. The absence of $n=1 \mathrm{~dB}_{\theta} / \mathrm{dt}$ signals from the Mirnov probes indicates that the RWM is stationary with respect to the wall from its onset. In contrast, at the time of the LTM onset the plasma rotation is already essentially zero over the entire minor radius. For this case the CER and Mirnov data show that before locking, the $n=1$ mode grows while rotating and slows down with the plasma.

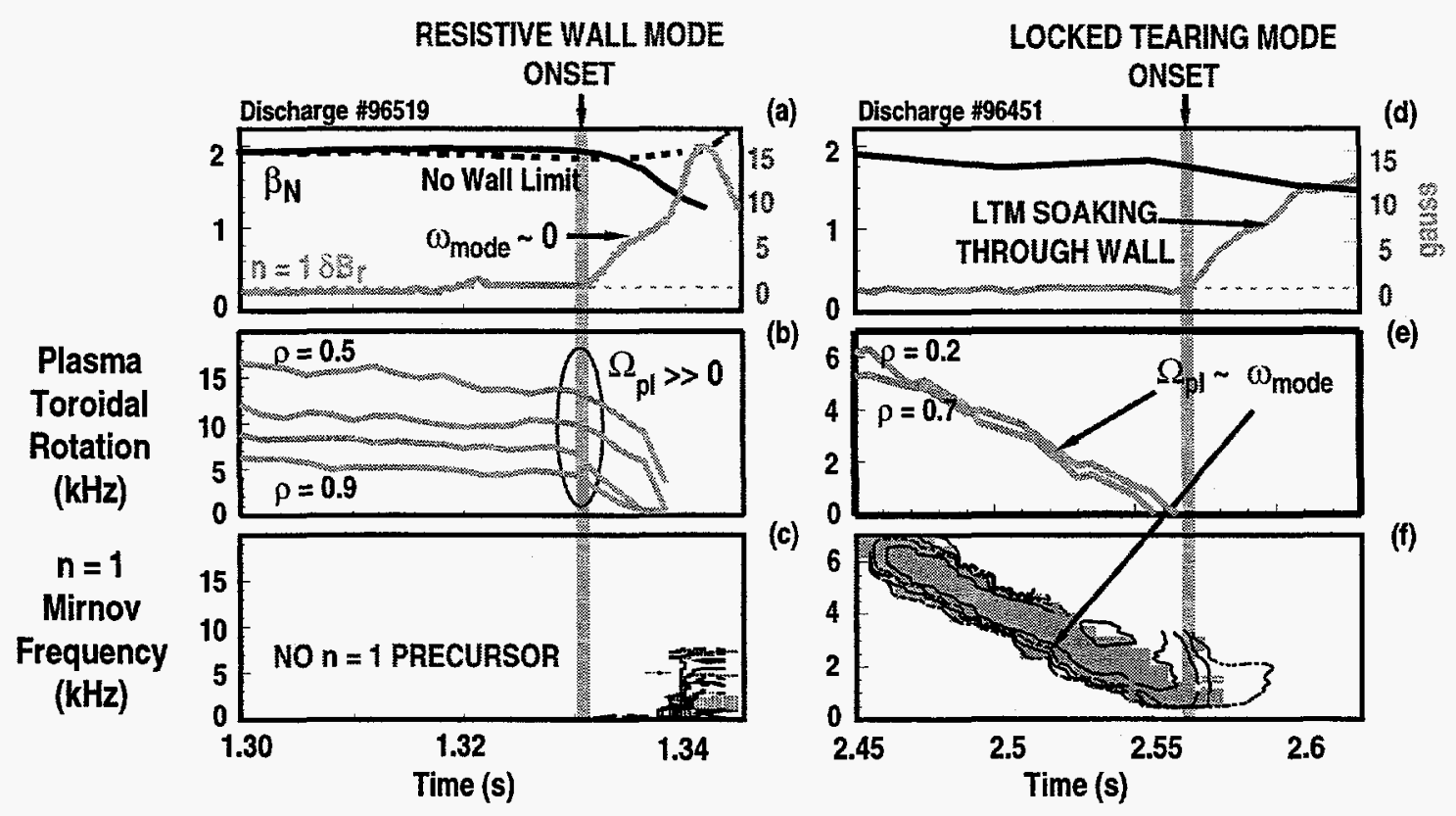

Fig. 5. Comparison of typical discharges with a resistive wall mode (a)-(c) (discharge \#96519) and with a tearing locked mode (d)-(f) (discharge \#96451). Time evolution of (a),(d) normalized beta and $n=1 B_{r}$ amplitude measured outside the vessel; and (b),(e) plasma rotation frequency at several radial locations. (c),(f) Contour plot of the $n=1$ Mirnov probe signals versus time and frequency.

Internal measurements of the mode structure from the ECE diagnostic confirm that the mode of Figs. 5(a)-(c) is not a tearing mode. Figure 6 shows a comparison of measured and predicted radial profile of the electron temperature change caused by the $n=1$ mode. The 


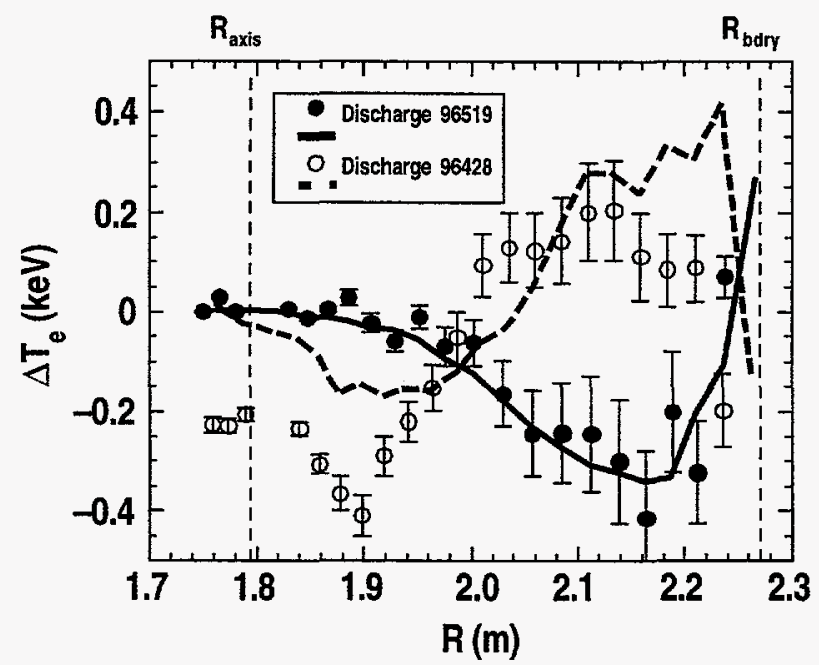

Fig. 6. Comparison of the radial profiles of the measured (solid circle data points) and predicted (solid curve) perturbed ECE $\mathrm{T}_{\mathrm{e}}$ signal for discharge \#96519 at $1338 \mathrm{~ms}$. Also shown is the data (open square data points) and prediction for a similar discharge \#96428 in which the mode has a $110^{\circ}$ phase difference from that for discharge \#96519 according to the toroidal saddle loop array. The prediction for discharge \#96428 is obtained by applying this phase difference to the instability for discharge \#96519. The magnetic axis at $R \sim 1.70 \mathrm{~m}$ and the plasma edge at $\mathrm{R} \sim 2.25 \mathrm{~m}$ are indicated.

measured change $\Delta \mathrm{T}_{\mathrm{e}}^{\mathrm{m}}$ is evaluated during time periods when, based on indications from magnetics and soft $\mathrm{x}$-rays, only an $\mathrm{n}=1$ perturbation is present. The predicted change, $\Delta \mathrm{T}_{\mathrm{e}}^{\mathrm{p}}$, is evaluated assuming that the mode responsible is the ideal plasma kink mode calculated by the GATO code in absence of a wall, and that the $T_{e}$ profile is convected with the mode displacement $\xi$ predicted along the $\mathrm{ECE}$ viewing line; i.e., $\Delta \mathrm{T}_{\mathrm{e}}^{\mathrm{p}}=\xi \cdot \mathrm{dT}_{\mathrm{e}} / \mathrm{dR}$. The toroidal phase of the GATO displacement is chosen according to the toroidal phase of the mode determined from magnetic diagnostics. Examples with two different toroidal phases of the mode are shown in Fig. 6. The measured mode structure reproduces well the qualitative features of the stability calculation, showing a radially extended structure whose sign changes appropriately with the magnetic phase of the mode. 


\section{Active Control of the Resistive Wall Mode}

The existence of a critical rotation frequency for stabilization suggests sustainment of the plasma rotation as one possible means for long time-scale stabilization of the RWM. However, the observation of a rotation slowdown in plasmas exceeding the no wall limit seems to imply that active control of the plasma rotation is required to maintain rotational stabilization. This may be achieved, for example, using rotating phased magnetic perturbations from coils internal to the vessel.

The alternative option is to feedback control the RWM using coils external to the conducting wall. This problem has been shown to be formally similar to the problem of active feedback of the axisymmetric vertical position instability. ${ }^{18}$ Active feedback stabilization of the axisymmetric mode is routinely used in DIII-D. ${ }^{19}$ Several algorithms for feedback control of the RWM have been proposed, including the "smart shell" 4 which uses externally applied magnetic perturbation to keep a zero net radial field at the wall, simulating the response of a perfectly conducting wall, and the "fake rotating shell," 20 where a phase shift is applied to the external magnetic perturbation to reproduce the leading phase shift response that the plasma would experience if the resistive wall were rotating toroidally.

Experimental tests have begun in DII-D and HBT-EP using the "smart shell" approach to stabilize the RWM. Experiments in DIII-D use the existing C-coil, which consists of six coils toroidally distributed at the midplane. In HBT-EP the conducting wall, internal to the vessel, is segmented to allow variation of the plasma wall separation. Recently, 10 of the original 20 aluminum wall segments have been replaced with stainless steel segments, so that also the wall time constant can now be effectively varied by inserting or retracting segments of different resistivity. Each stainless steel segment is equipped on the vacuum side with three active coils.

The 3D electromagnetic code VALEN has been used to predict the efficiency of the feedback systems in DIII-D and HBT-EP. The VALEN code integrates a 3D finite element electromagnetic representation of vessel, sensors, coils, and plasma, a plasma instability modeled by equivalent helical current distribution, and the feedback control logic. The VALEN results in Fig. 7 show that feedback stabilization is possible in DIII-D using the existing coils. The results of the figure, applied to a plasma with $\beta_{\mathrm{N}}^{\text {nowall }}=3$ and $\beta_{\mathrm{N}}^{\text {ideal wall }}=5$ promise an improvement of about $15 \%$ in the beta limit with active feedback using the existing $\mathrm{C}$-coils. A greater increase of the beta limit is predicted with the addition of coils above and below the existing C-coil.

Preliminary experiments in DIII-D using open-loop active control show encouraging results. A series of discharges with reproducible RWM onset and toroidal phase were run, but one discharge included a static $n=1$ magnetic perturbation which was programmed to 


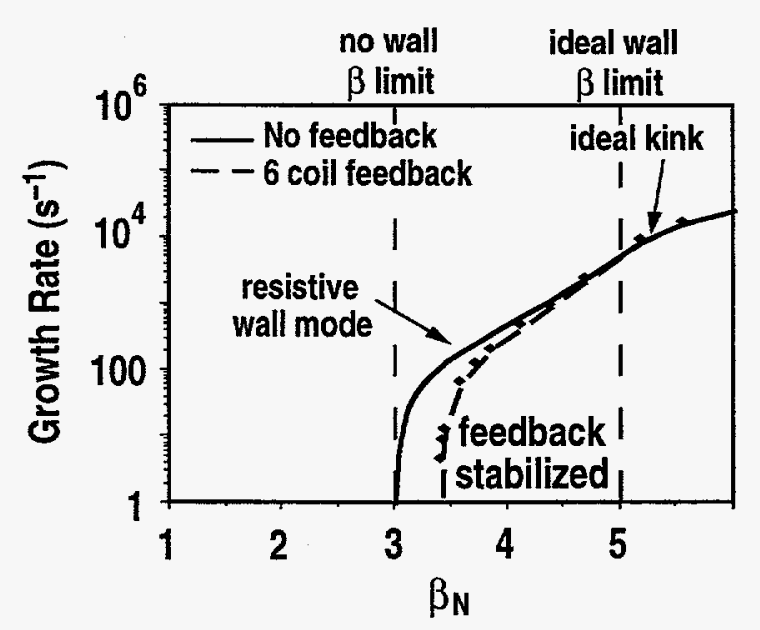

Fig. 7. Growth rate of the instability for a DIII-D plasma above the no-wall $\beta_{\mathrm{N}}$ limit versus instability drive strength predicted by the VALEN 3D code. Different symbols denote results for the cases without feedback (hollow squares); with feedback using the existing coils (solid diamonds); and with feedback using an extended coil set (hollow diamonds).

step on at the time of the RWM onset with a phase opposing the mode. At the time of the expected RWM onset, the plasma rotation, $T_{e}$, and $\beta_{N}$ start to decrease rapidly in discharges without the $n=1$ opposing field, while they hesitate and then recover in the open loop discharge as the amplitude of the external field increases. Measurements of the $n=1$ mode amplitude, after correction for the applied perturbation and its induced currents, indicate that the RWM onset was postponed for greater than $20 \mathrm{~ms}$.

A broad survey of active mode feedback in HBT-EP using a 30-coil smart shell with variable wall time constant has started. The results will allow the benchmark of the quantitative feedback control model VALEN, which could then be used as a tool for the design of active mode control system on a broad class of toroidal devices. 


\section{Summary and Discussion}

New techniques and improved diagnostic capabilities have greatly extended the duration, beta enhancement factor, and reproducibility of wall-stabilized discharges in DII-D, and have allowed the study of previously inaccessible resistive wall mode physics. The RWM has now been directly identified from external magnetic and internal diagnostic measurements. Reproducible measurements of the critical plasma rotation for stabilization of the RWM can now be compared quantitatively with the various theoretical predictions. A slowing of the plasma rotation is shown to be correlated with $\beta_{\mathrm{N}}$ exceeding $\beta_{\mathrm{N}}^{\text {no wall }}$ and can persist even when there is no discernible MHD activity, suggesting the need for a better physics understanding of the momentum exchange between the plasma and wall when $E_{\mathrm{w}}>1$. The experimental results suggest that for stabilization of the RWM by plasma rotation, active control of the plasma rotation might be needed. Alternatively, RWM stabilization is predicted to be possible in DIII-D with active feedback using the existing error field correction coils, and preliminary experiments show encouraging results. Active control of the angular momentum profile and active feedback control of the RWM hold great promise for substantially improving the stability of high $\beta$ advanced tokamak plasmas. 


\section{Acknowledgments}

This is a report of research sponsored by the U.S. Department of Energy under Grants DE-FG02-89ER53297 and DE-FG03-97ER54415, and Contracts DE-AC03-89ER51114, DE-AC02-76CH03073, DE-AC05-96OR22464, and W-7405-ENG-48. 


\section{References}

${ }^{1}$ E.J. Strait, T.S. Taylor, A.D. Turnbull, J.R. Ferron, L.L. Lao, B.W. Rice, O. Sauter, S.J. Thomson, and D. Wròblewski, Phys. Rev. Lett. 74, 2483 (1994).

${ }^{2}$ M. Okabayashi, N. Pomphrey, J. Manickam, D.J. Ward, R.E. Bell, R.E. Hatcher, R. Kaita, F.M. Levinton, D.W. Roberts, S. Sesnic, Y. Sun, and H. Takahashi, Nucl. Fusion 36, 1167 (1996).

${ }^{3}$ A.M. Garofalo et al., Nucl. Fusion 38, 1029 (1998).

${ }^{4}$ C.M. Bishop, Plasma Phys. Contr. Fusion 31 (1989) 1179.

${ }^{5}$ A.D. Turnbull, T.S. Taylor, Y.R. Lin-Liu, and H. St. John, Phys. Rev. Lett. 74, 718 (1995).

${ }^{6}$ C. Kessel, J. Manickam, G. Rewoldt, and W.M. Tang, Phys. Rev. Lett. 72, 1212 (1994).

${ }^{7}$ A.D. Turnbull, T.S. Taylor, E.J. Strait, S.J. Thompson, M.S. Chu, J.R. Ferron, R.J. La Haye, L.L. Lao, R.T. Snider, et al., in Plasma Phys. and Contr. Nucl. Fusion Res. (Proc. 15th Int. Conf. Seville, 1994) (IAEA, Vienna, 1995) Vol. 1, p. 705.

${ }^{8}$ T.S. Taylor, E.J. Strait, L. L. Lao, M.E. Mauel, A.D. Turnbull, K.H. Burrell, M.S. Chu, J.R. Ferron, and R.J. Groebner, Phys. Plasmas 2, 2890 (1995).

${ }^{9}$ T.H. Ivers, E. Eisner, A. Garofalo, R. Kombargi, M.E. Mauel, D. Maurer, D. Nadle, G.A. Navratil, M.K.V. Sankar, M. Su, E. Taylor, et al., Phys. Plasmas 3, 1746 (1996).

${ }^{10}$ A. Bondeson and D.J. Ward, Phys. Rev. Lett. 72, 2709 (1994).

${ }^{11}$ R. Betti and J.P. Freidberg, Phys. Rev. Lett. 74, 2949 (1995).

12A.H. Boozer, Phys. Plasmas 2, 4521 (1995).

13J.M. Finn, Phys. Plasmas 2, 3782 (1995).

${ }^{14}$ L.C. Bernard, F.J. Helton, and R.W. Moore, Comput. Phys. Commun. 21, 377 (1981).

${ }^{15}$ A. Garofalo, J. Bialek, M.S. Chu, E.D. Fredrickson, R.J. Groebner, R.J. La Haye, L.L.

Lao, G.A. Navratil, B.W. Rice, and S.A. Sabbaugh, EPS Proceedings 1998.

16R. Betti, Phys. Plasmas 5, 3615 (1998).

${ }^{17}$ C.G. Gimblett, Nucl Fusion 26, 617 (1986).

${ }^{18}$ A.H. Boozer, Phys. Plasmas 5, 3350 (1998).

${ }^{19}$ E.A. Lazarus et al., Phys. Fluids B 3, 2220 (1991).

${ }^{20} \mathrm{R}$. Fitzpatrick and T.H. Jensen, Phys. Plasmas 3, 2641 (1996). 